\title{
A simple analysis system for the estimation of recombination efficiency using fluorescence-activated cell sorting
}

\author{
Min Soo Kim, Won Hee Kim, Gyun Min Lee* \\ Department of Biological Sciences, Korea Advanced Institute of Science and Technology, 373-1 Kusong-Dong, \\ Yusong-Gu, Daejon 305-701, Republic of Korea
}

Received 30 March 2006; received in revised form 4 June 2006; accepted 24 July 2006

\begin{abstract}
A simple and accurate analysis system for the estimation of recombination efficiency in vivo using fluorescence-activated cell sorting (FACS) was designed and was subsequently used to compare the efficiency of recombination related to different spacer mutants. $\mathrm{F}_{3}$ and $\mathrm{F}_{5}$ mutant sequences were used for Flpe-mediated cassette exchange, and $m 2$ and lox2272 mutant sequences were used for Cre-mediated cassette exchange due to their high incompatibility with wild-type sequences. The incompatibilities with wild-type were almost the same between mutant sequences. However, the recombination efficiencies were different. $\mathrm{F}_{3}$ and $m 2$ could mediate more efficient recombination than $\mathrm{F}_{5}$ and lox2272, respectively. These results are consistent with the fact that the sequence of spacer region affects not only the reactivity upon wild-type sequence but also the recombination efficiency. It was also confirmed that the recombination process was mediated in a site-specific manner through PCR analysis using different sizes of exchange cassettes. In this experiment, the feasibility of the FACS analysis system for the estimation of recombination efficiency was verified. This system should be readily applicable for estimating recombination efficiency of other various mutant candidates, which will contribute to more precise and efficient site-specific recombination.
\end{abstract}

(c) 2006 Elsevier B.V. All rights reserved.

Keywords: Site-specific recombinase; Recombinase-mediated cassette exchange; Incompatibility; Recombination efficiency; FACS

\section{Introduction}

The random integration of foreign genes into a chromosome during typical transfection leads to unpredictable gene expression. Actually, most transgenes are repressed in the chromosome in a

\footnotetext{
* Corresponding author. Tel.: +82 428692618 ; fax: +82428692610 .

E-mail address: gyunminlee@kaist.ac.kr (G.M. Lee).
}

position-dependent manner (Festenstein et al., 1996). The importance of the chromosomal site of integration is more prominent during long term culture. Transgene expression can be silenced if the gene is integrated near the heterochromatic part of the chromosome (Bell and Felsenfeld, 1999).

To circumvent this position effect, the use of site-specific recombinases has been applied. Cre from bacteriophage P1 (Hoess et al., 1982), Flp from Saccharomyces cerevisiae (McLeod et al., 1986), and 
its thermostable variant, Flpe (Buchholz et al., 1998) are widely used recombinases. Targeted integration of transgenes into predefined chromosomal loci by these site-specific recombinases can lead to more predictable and elevated transgene expression. For example, a hot-spot on a chromosome, which allows for high-level expression from a single or only a few gene copies, can be targeted reproducibly for high-level expression of any transgene by site-specific recombination. With this approach, the tedious and time-consuming cell line development process, which occurs with traditional random integration, can be improved.

Among various recombination strategies, it is believed that recombinase-mediated cassette exchange (RMCE) is one of the most promising strategies for the targeted integration of transgenes. RMCE guarantees effective recombination and excludes unnecessary vector sequences of prokaryotic origin which can inhibit transgene expression by methylation-dependent inactivation (Baer and Bode, 2001).

For stable integration of a transgene by the RMCE strategy, mutant recognition sequences, which are incompatible with wild-type sequence, are indispensable, as in general recombination strategies. For the past decade, much work concerning mutant sequence has been performed. The induction of mutation in inverted repeat regions of a wild-type recognition sequence increased the recombination efficiency by decreasing a reverse reaction or an excision (Albert et al., 1995). However, the binding affinity of DNA against recombinase can be impaired in this system. Knowledge concerning the role of nucleotide sequences within the FRT spacer region and the lox $\mathrm{P}$ spacer region led to the use of a mutant with a mutation in its spacer region (Umlauf and Cox, 1988; Lee and Jayaram, 1995; Lee and Saito, 1998). This promoted the recombination process by decreased reactivity upon wild-type recognition sequence and intact binding affinity against recombinase. Among various mutant sequences, it seems that lox2272 (Lee and Saito, 1998) and $m 2$ (Langer et al., 2002) in the Cre/loxP system, $F_{3}$ and $F_{5}$ (Schlake and Bode, 1994) in the Flpe/FRT system have the lowest reactivity upon a wild-type sequence (Branda and Dymecki, 2004).

Despite much work for the identification of novel mutants, studies on the efficiency of recombination related to these mutants in vivo were insufficient. Therefore, it will be meaningful to investigate whether these mutant sequences can contribute to effective sitespecific recombination and to compare the efficiency of the recombination related to different mutants. A similar approach was performed by Kolot et al. on the coliphage HK022 integrase system (Kolot et al., 2003). However, we have focused on the recombination events mediated by the RMCE strategy, which is one of the most powerful tools for gene targeting, and applied the most widely used recombinases (Cre and Flpe). Here, we present a simple and accurate analysis system for the estimation of recombination efficiency in vivo using FACS.

\section{Materials and methods}

\subsection{Plasmid construction}

\subsection{1. pbleo family vectors}

pIRESbleo-MCS was obtained by slight modification of pIRESbleo (BD Biosciences, Franklin Lakes, NJ). EGFP coding region was obtained from pIRES2-EGFP (BD Biosciences) by PCR amplification. The primers used for this PCR reaction were 5'-GAATTCATGGTGAGCAAGGGCGAGGA-3' and 5'-GAATTCGATCTAGAGTCGCGGCCGCT-3'. The EGFP coding region was cloned into the EcoRI site of the pIRESbleo-MCS vector, resulting in pbleo-EGFP. The EGFP gene can then be expressed by the CMV promoter which pre-exists in pIRESbleo-MCS.

pbleo-EGFP-lox $\mathrm{P}$ and pbleo-EGFP-FRT were prepared by inserting synthesized lox $\mathrm{P}$ and $F R T$ fragments between NotI and SacII sites of pbleoEGFP. Subsequent ligation of HindIII-BamHI fragments of various mutants and wild-type sequences resulted in pbleo-loxP-EGFP-loxP, pbleo- $m 2$-EGFPloxP, pbleo-lox2272-EGFP-loxP, pbleo-FRT-EGFP$F R T$, pbleo-F 3 -EGFP- $F R T$, and pbleo-F5-EGFP-FRT, respectively. These re-iterated constructs were referred to as pbleo family vectors (Fig. 1), which were used to investigate the incompatibilities of various spacer mutants. Incompatibility is defined as the ability of a recognition sequence to discriminate against recombination with another recognition sequence (Langer et al., 2002). A high level of incompatibility with wild-type sequence is an important factor to consider when identifying mutants for efficient recombination. Each vector has an EGFP gene for monitoring the level of green 


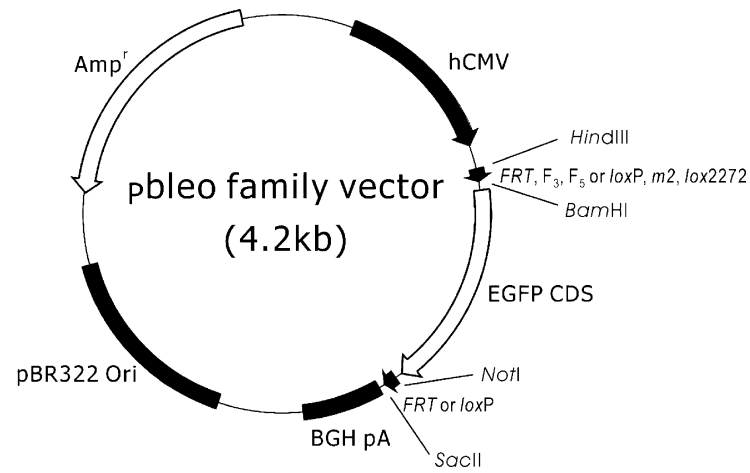

Fig. 1. Schematic map of the pbleo family vector used for the incompatibility test. hCMV, human cytomegalovirus promoter; BGH pA, bovine growth hormone poly A addition signal; EGFP CDS, coding region of EGFP gene; $\mathrm{Amp}^{\mathrm{r}}$, ampicillin-resistant gene; $\mathrm{pBR} 322$ Ori, pBR322 replication origin. The EGFP CDS is flanked by wild-type sequence or mutant sequences and their corresponding wild-type sequence.

fluorescence which disappears when an excision reaction occurs due to the reactivity between recognition sequences. The pbleo family vector can be divided into two DNA constructs under the expression of recombinase if reactivity exists between the two recognition sequences. With this design, higher reactivity resulted in decreased fluorescence (Fig. 2).

\subsection{2. pIC family vectors}

pIRESbleo was digested by AatII, and self-ligated to remove the CMV promoter completely. The resulting vector was designated as pIC. The EGFP coding region, which was also used to construct pbleo family
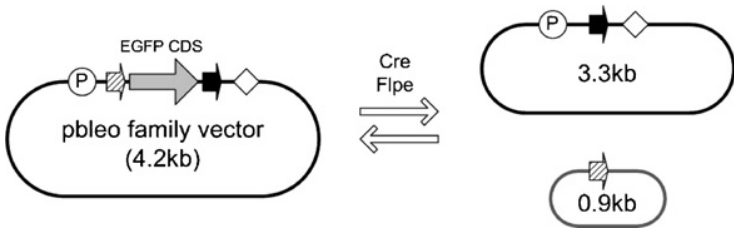

Fig. 2. Possible reaction in the incompatibility test. The EGFP CDS is flanked by wild-type sequence or mutant sequences $(\otimes)$ and their corresponding wild-type sequence $(\rightarrow)$. The EGFP CDS can be expressed by the CMV promoter (D) and the polyadenylation signal $(\diamond)$. The pbleo family vector can be divided into two DNA constructs under the expression of recombinase if reactivity exists between two recognition sequences. The EGFP CDS can then no longer be expressed. Therefore, the decrease of green fluorescence represents the extent of reactivity between two recognition sequences. vector, was cloned into the EcoRI site of the pIC vector, resulting in pIC-EGFP.

pIC-EGFP-loxP and pIC-EGFP- $F R T$ were prepared by inserting synthesized lox $\mathrm{P}$ and $F R T$ fragments between NotI and SacII sites of pIC-EGFP.

Plasmids containing lox $\mathrm{P}$ mutants $(\mathrm{m} 2$ and lox2272), loxP, $F R T$ mutants $\left(\mathrm{F}_{3}\right.$ and $\left.\mathrm{F}_{5}\right)$, and $F R T$ were derived by ligating synthesized HindIII-BamHI fragments, which were designated as pIC-m2-EGFP-loxP, pIC-lox2272-EGFP-loxP, pIC-loxP-EGFP-loxP, pIC$\mathrm{F}_{3}$-EGFP-FRT, pIC-F5-EGFP-FRT, and pIC-FRTEGFP-FRT, respectively. These re-iterated constructs were designated as pIC family vectors. Fig. 4 presents the schematic map of the pIC family vector.

\subsection{3. pDC family vectors}

The overall processes of $\mathrm{pDC}$ family vector construction were similar to those of pIC family vector construction. $\mathrm{pDC}$ was obtained by slight modification of the multicloning site of pCVM-dhfr2 (Aprogen, Daejon, Korea). HSV-TK gene, which functions merely as a spacer in this experiment, was isolated as the EcoRI fragment from $\mathrm{pPNT}$ and cloned into the corresponding site $(E c o \mathrm{RI})$ of $\mathrm{pDC}$, resulting in $\mathrm{pDC}-\mathrm{TK}$.

Ligation of loxP and $F R T$ fragments flanked by $N o t \mathrm{I}$ and SacII produced pDC-TK-loxP and pDC-TK-FRT. Subsequent ligation of HindIII-BamHI fragments of various mutants and wild-type recognition sequences resulted in pDC-m2-TK-loxP, pDC-lox2272-TK-loxP, pDC-loxP-TK-loxP, pDC-F $3-\mathrm{TK}-F R T$, pDC-F5-TK$F R T$, and pDC-FRT-TK-FRT, respectively. These reiterated constructs were referred to as $\mathrm{pDC}$ family vectors. The schematic map of the $\mathrm{pDC}$ family vector is shown in Fig. 4. The forward and reverse PCR primers for the PCR experiment performed later have an exact homology only with the $\mathrm{pDC}$ family vector sequences.

pIC family vectors and pDC family vectors were used for the estimation of recombination efficiency. The level of green fluorescence, which increases when the EGFP coding region is properly located behind the pre-existing CMV promoter after precise cassette exchange, represents the efficiency of the recombination mediated in a site-specific manner (Fig. 5).

\subsubsection{Recombinase expression vectors}

The Cre and Flpe genes were kindly provided by Dr. S.C. Kim, KAIST and Dr. F. Stewart, EMBL, respectively (Yoon et al., 1998; Buchholz et al., 1998). A 
mammalian expression vector, pEGFP-C1 (BD Biosciences) was used for the construction of Cre and Flpe expression vectors. pEGFP-C1 contains a CMV promoter for driving Cre and Flpe expression. pEGFP-C1 was digested by AgeI and XmaI to remove the EGFP gene. After digestion, the linearized DNA was selfligated, resulting in $\mathrm{p} \triangle \mathrm{EGFP}-\mathrm{C} 1$. $\mathrm{p} \triangle \mathrm{EGFP}-\mathrm{C} 1$ was digested by NheI and $M l u \mathrm{I}$. Then, Cre and Flpe gene were cloned into the corresponding sites (NheI and $M l u \mathrm{I})$ of $\mathrm{p} \Delta \mathrm{EGFP}-\mathrm{C} 1$, resulting in $\mathrm{p} \Delta \mathrm{EGFP}-\mathrm{Cre}$ and $\mathrm{p} \triangle \mathrm{EGFP}-\mathrm{Flpe}$, respectively.

\subsubsection{Positive control vector}

pIC-F 3 -EGFP-FRT was digested by HindIII and SacII. A fragment of about $0.9 \mathrm{~kb}$ which includes EGFP $\mathrm{CDS}, \mathrm{F}_{3}$, and $F R T$ was cloned into the corresponding sites (HindIII and SacII) of pDC-F $3-T K-F R T$, resulting in $\mathrm{pDC}-\mathrm{F}_{3}-\mathrm{EGFP}-F R T$. pDC-F 3 -EGFP- $F R T$ can function as a positive control because its structure is an exact match with one of the plasmids after precise RMCE. The EGFP gene can then be expressed well under the control of a CMV promoter.

The nucleotide sequences at the sites of insertion were verified by DNA sequencing. All plasmids were constructed using standard techniques, and details of their construction are available upon request.

\subsection{Cell culture}

293 T cells (human embryonic kidney cell, ATCC CRL-11268) were used for transfection. The culture medium used was Dulbecco's modified Eagle medium (DMEM; Invitrogen, Grand Island, NY) supplemented with $10 \%$ (v/v) fetal bovine serum (FBS, JRH Biosciences, Lenexa, KS). Cells were maintained as monolayer cultures in $25-\mathrm{cm}^{2}$ T-flasks in a $5 \% \mathrm{CO}_{2} /$ air mixture, humidified at $37^{\circ} \mathrm{C}$. Upon reaching confluency, they were diluted 1:10 with fresh medium every 3 days.

\subsection{Transient transfection}

One day prior to transfection, 293T cells were seeded at a density of $10^{5}$ cells $/ \mathrm{ml}$. Cells were transfected with two combinations of plasmids simultaneously for the incompatibility test. One combination of plasmids consisted of only pbleo family vectors. The other was a mixture of pbleo family vectors with a cor- responding recombinase expression vector. The molar ratio of pbleo family vectors and recombinase expression vectors was $1: 2$. The amount of pbleo family vector was $2 \mu \mathrm{g}$.

In the case of the recombination efficiency test, cells were transfected with four combinations of plasmids at the same time using Lipofectamine ${ }^{\mathrm{TM}} 2000$ (Invitrogen) following the manufacturer's recommendation. The four combinations of plasmids consisted of a negative control (only Lipofectamine was added), a positive control (pDC-F 3 -EGFP-FRT), a mixture of pIC family vectors and $\mathrm{pDC}$ family vectors, and a mixture of pIC family vectors, $\mathrm{pDC}$ family vectors, and recombinase expression vectors. To minimize fluorescence disappearance caused by a reverse reaction, and at the same time, to minimize an interaction between pIC family vectors, the molar concentration $(\mathrm{M})$ of $\mathrm{pDC}$ family vector was adjusted to be much higher than that of pIC family vector. The molar ratio of pIC family vectors, pDC family vectors, and recombinase expression vectors was 1:5:3. The amount of pIC family vector was $0.5 \mu \mathrm{g}$.

In both experiments, the total amount of DNA was adjusted to the same concentration with that of the reaction, which has the maximum amount of DNA due to the fact that the total amount of DNA can affect transfection efficiency of individual reactions. $\mathrm{p} \triangle \mathrm{EGFP}-\mathrm{C} 1$ was used for the adjustment of DNA.

\subsection{Fluorescence-activated cell sorting (FACS) analysis}

Half of the cells from each flask were harvested and washed with PBS $48 \mathrm{~h}$ after transfection. The FACSCalibur $^{\text {TM }}$ system (Becton Dickinson, San Jose, CA) was used for analyzing the samples prepared. The instrumental settings are summarized previously (Park and Lee, 2000). Ten thousand cells were analyzed for each sample. Data analysis was performed using the WinMDI 2.8 program.

\subsection{PCR analysis}

For PCR analysis, extrachromosomal DNA from the remaining half of the transfected cells was extracted using a modified version of the Hirt procedure (Arad, 1998). The primers used for the PCR reaction were 5'-CGACTCACTATAGGGAGACC-3' and 
$5^{\prime}$-ATAGAATAGGGCCCTCTAGC-3'. These primers were annealed at $56^{\circ} \mathrm{C}$ and elongated for 2 or $5 \mathrm{~min}$.

\section{Results and discussion}

\subsection{Validation of constructed vectors}

Many kinds of vectors were constructed in this study. Among them, pIC family vectors, which have a promoterless EGFP gene, were designed to have no green fluorescence. pDC family vectors and recombinase expression vectors also must not expose any green fluorescence. We validated that these vectors have approximately the same fluorescence level with the negative control by FACS analysis (data not shown). Therefore, it was confirmed that there were no factors present that might distort the result of FACS analysis.

\subsection{Incompatibility test of various spacer mutants}

$\mathrm{F}_{3}$ and $\mathrm{F}_{5}$ mutant sequences for Flpe-mediated cassette exchange were selected, and $m 2$ and lox 2272 mutant sequences for Cre-mediated cassette exchange were selected as candidate sequences for evaluating our FACS analysis system due to their high levels of incompatibility with wild-type sequences. To confirm incompatibilities of these mutant sequences, pbleo family vectors were constructed (Fig. 1). As described in Section 2, the incompatibility test was performed using the strategy in which higher reactivity between recognition sequences resulted in decreased fluorescence (Fig. 2).

As shown in Fig. 3, reactions transfected with only pbleo family vectors had similar levels of fluorescence in each recombinase system due to the same transfection efficiency. However, the changing patterns of fluorescence level were different among recognition sequences when recombinase expression vectors were also applied. Under the expression of Flpe recombinase, many pbleo-FRT-EGFP-FRT plasmids lost their fluorescence due to an excision reaction between two identical recognition targets. To estimate the extent of incompatibility, the total amount of fluorescence in the selected region where cells have a positive green fluorescence was calculated after taking into account the number of cells and the mean fluorescence value of that region. The total amount of fluorescence in a mixture of pbleo- $F R T$-EGFP- $F R T$ and $\mathrm{p} \triangle \mathrm{EGFP}-\mathrm{Flpe}$ was $23 \%$ compared to that of only pbleo-FRT-EGFP-FRT. The level of incompatibility between two identical $F R T$ sequences was significantly low as was expected. Two plasmids that result after an excision reaction can also serve as substrates for Flpe recombinase. However, for the reason that an intramolecular reaction is more likely to occur than an intermolecular reaction, the value falls between $0 \%$ and $50 \%$. In contrast, the fluorescence lev-

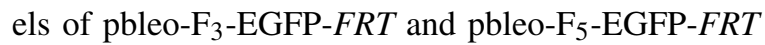
under the expression of Flpe recombinase were much higher than that of pbleo-FRT-EGFP-FRT. Those were $80 \%$ and $89 \%$ compared to their corresponding controls which are deficient in the recombinase expression vector. Such a high level of fluorescence indicates that $F_{3}$ and $\mathrm{F}_{5}$ mutant sequences have very low reactivity with the FRT sequence. These results are exactly consistent with the previous work by Schlake and Bode (1994), which supports the feasibility of our FACS analysis system for further work, the estimation of recombination efficiency. Consequently, it was clarified that $\mathrm{F}_{5}$ has almost absolute incompatibility with wild-type $F R T$ sequence and $\mathrm{F}_{3}$ also has a high level of incompatibility. Therefore, $F_{3}$ and $F_{5}$ may be good mutant candidates for an efficient site-specific recombination.

The result from an incompatibility test in the Cremediated cassette exchange system was quite different from that of the Flpe-mediated cassette exchange system. The total amount of fluorescence in a mixture of pbleo-loxP-EGFP-loxP and $\mathrm{p} \Delta$ EGFP-Cre was only $1 \%$ in comparison with a control reaction. It was reported that Cre and Flpe recombinases have slight differences in their kinetic characteristics (Ringrose et al., 1998). Cre binds its target site more strongly and with higher cooperativity than Flpe. Cre also has a highly more stable synaptic complex than Flpe. It is assumed that this stronger binding with its target site and higher synaptic stability of Cre over Flpe enabled almost complete excision of DNA substrates and resulted in extreme leaning of the equilibrium point to the right (Fig. 2). The fluorescence levels of pbleo-m2-EGFP-loxP and pbleo-lox2272-EGFP-loxP under the expression of Cre recombinase were $66 \%$ and $60 \%$ compared to their corresponding controls, which are deficient in the recombinase expression vector. It is assumed that the higher synaptic stability of Cre over Flpe provided a higher chance of unexpected recombination and resulted in a 

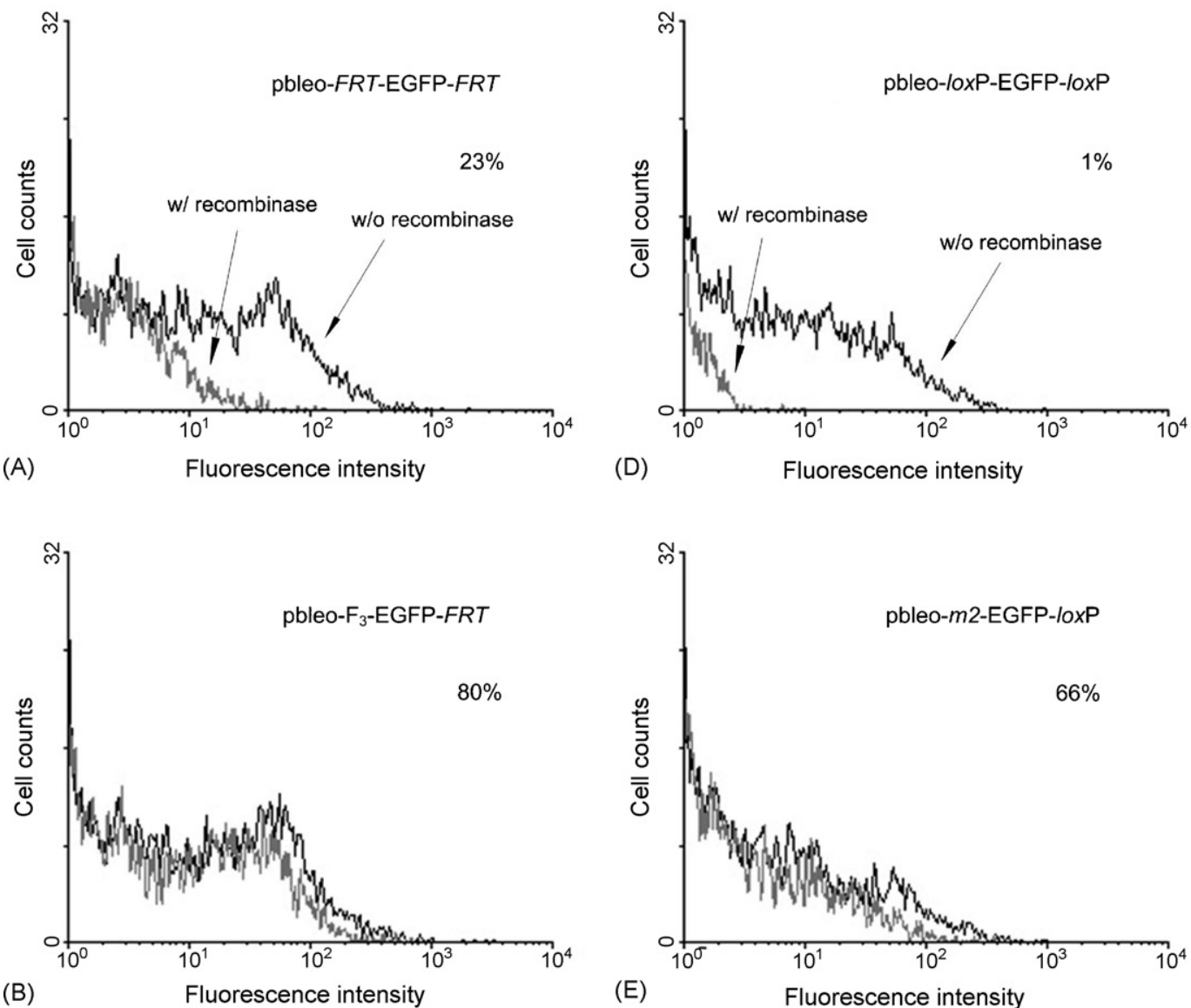

(E)

Fluorescence intensity
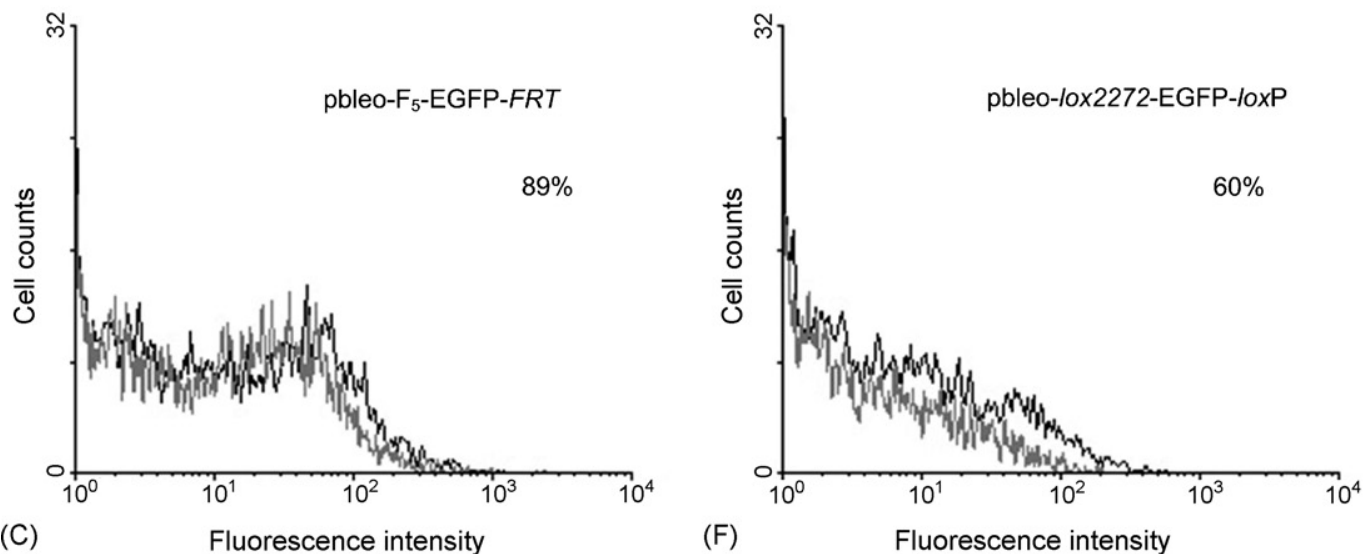

Fig. 3. FACS analysis of the incompatibility test. The ratio of fluorescence levels of each pbleo family vector with recombinase compared to those without recombinase, which served as positive controls, were measured (A-F). Incompatibility of recognition sequence with wild-type sequence increases as the fluorescence level approaches the control fluorescence level. 
slightly decreased incompatibility between mutant and wild-type sequence. However, the extents of incompatibility of $m 2$ and of lox2272 are still high, even if those do not satisfy the level proposed as almost absolute in previously published articles (Lee and Saito, 1998; Langer et al., 2002).

\subsection{Estimating efficiency of recombination related to various spacer mutants}

Previously, we showed that the incompatibilities with the wild-type recognition sequence were almost the same between spacer mutant sequences, which
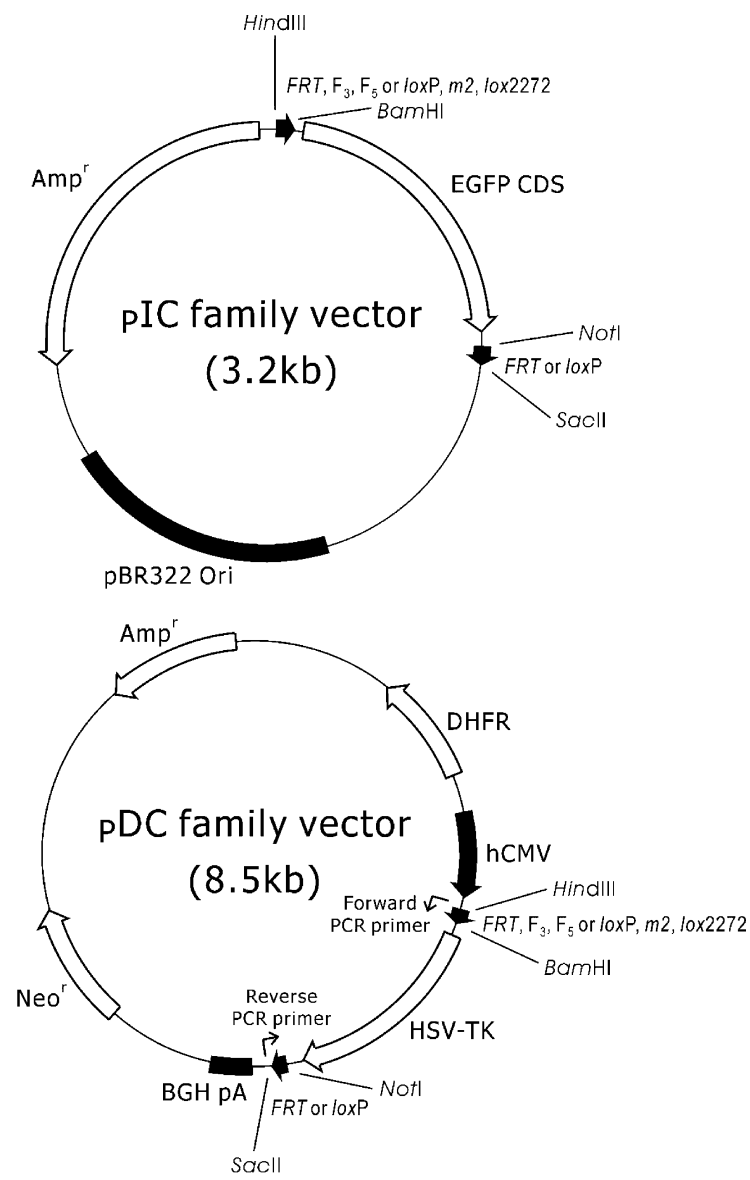

Fig. 4. Schematic map of the pIC family vector and the pDC family vector, which were used for the recombination efficiency test. HSV-TK, herpes simplex virus thymidine kinase; $\mathrm{Neo}^{\mathrm{r}}$, neomycinresistant gene. The forward and reverse PCR primers were designed to have an exact homology only with the pDC family vector sequences. had high levels of incompatibilities with the wild-type sequence. However, the high level of incompatibility is not the only precondition for an efficient site-specific recombination by RMCE since the sequence of the modified spacer itself can affect recombination efficiency (Lee and Saito, 1998). Therefore, a simple and accurate analysis system was designed to estimate the efficiency of recombination related to spacer mutant sequences in vivo using FACS.

pIC family vectors and pDC family vectors were used for the estimation of recombination efficiency
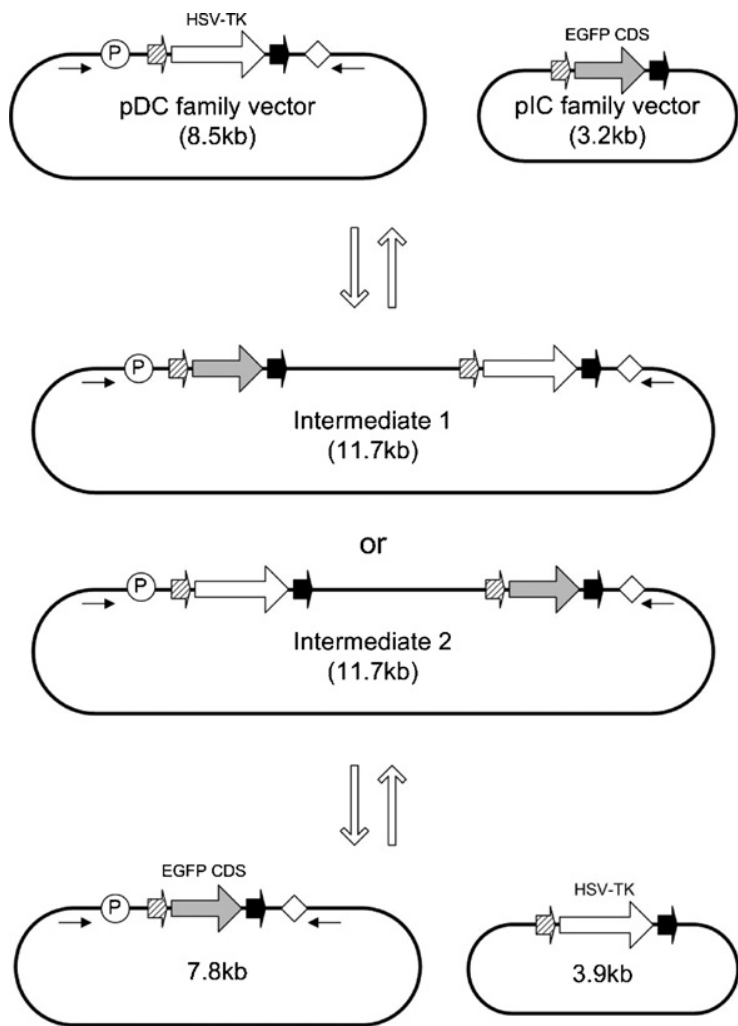

Fig. 5. Possible reactions in the recombination efficiency test. The EGFP CDS and the HSV-TK are flanked by wild-type sequence or mutant sequences $(\Leftrightarrow)$ and their corresponding wild-type sequence $(\Leftrightarrow)$. Two intermediate forms are possible. An additional reciprocal crossover leads to cassette exchange, which results in two new DNA constructs of 7.8 and $3.9 \mathrm{~kb}$, or return to the initial vectors (pDC family vector and pIC family vector). The EGFP CDS can not be expressed in the pIC family vector. However, it can be expressed by the CMV promoter of the pDC family vector after complete exchange. Therefore, the increasing level of green fluorescence represents the recombination efficiency. Arrows in vector constructs represent primers for PCR. 
(Fig. 4). The promoter-trap strategy was applied as described in Section 2. With this design, the level of green fluorescence represents the efficiency of the recombination mediated in a site-specific manner (Fig. 5).

Fig. 6 shows the result of FACS analysis concerning Cre-mediated cassette exchange. The fluorescence levels in the four combinations of plasmids (a negative control, a positive control vector, a mixture of corresponding pIC family vectors and pDC family vectors, and a mixture of corresponding pIC family vectors,
pDC family vectors, and a recombinase expression vector) were analyzed. Thereafter, the ratio of the upper region compared to the negative control, which represents a positive fluorescence, was calculated. The total amount of DNA in all reactions except the negative control was adjusted to the same concentration with that of the mixture of pIC family vectors, pDC family vectors, and a recombinase expression vector. This was done because the transfection efficiency may be changed according to the amount of DNA even if all other conditions are the same. The ratio of fluorescent

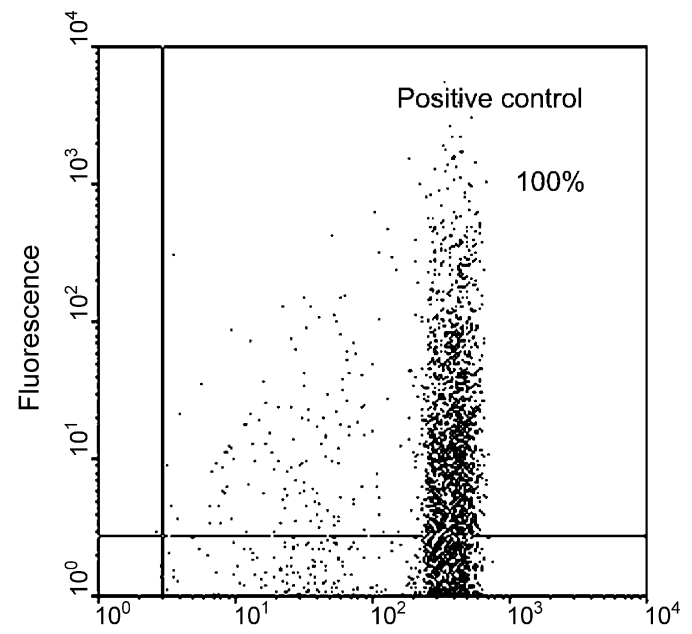

(A)

Cell size

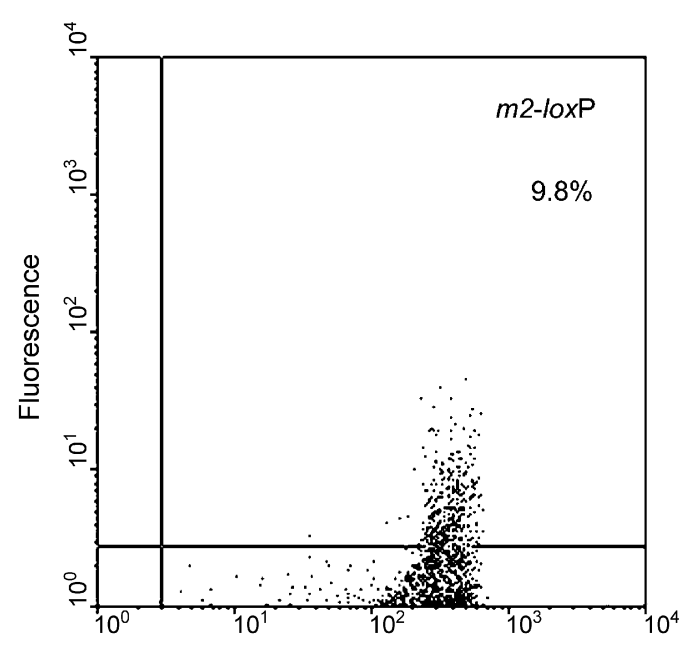

(C)

Cell size

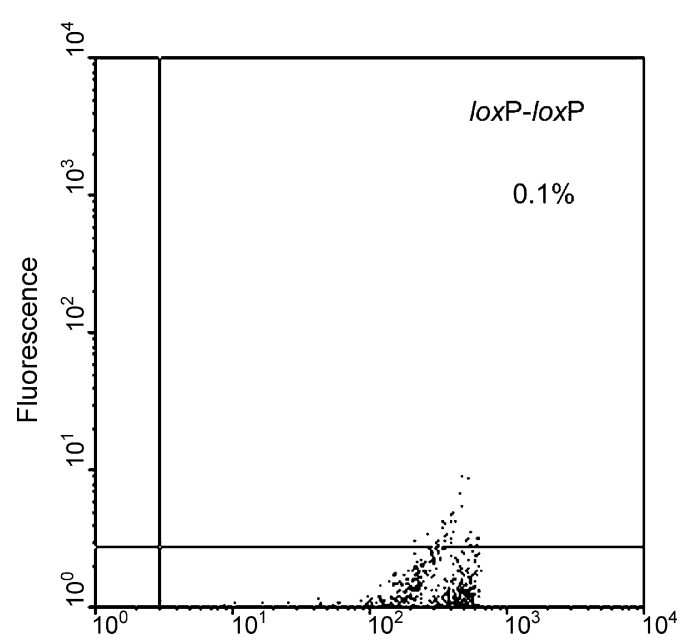

(B)

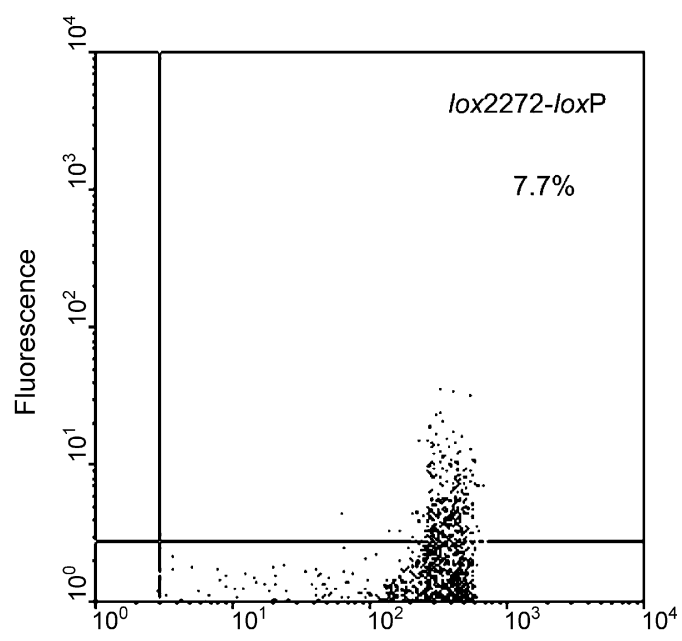

(D)

Cell size

Fig. 6. FACS analysis of the recombination efficiency test in the Cre-mediated cassette exchange. The ratio of total fluorescence of recombinations related to loxP (B), $m 2$ (C), and lox2272 (D) compared to that of the positive control (A) were estimated. 
cells in the positive control reaction was $17 \%$. There were no significant appearances of green fluorescence in the mixtures of $\mathrm{pIC}$ and $\mathrm{pDC}$ family vectors without a recombinase expression vector (data not shown). This implies that there was no unexpected DNA rearrangement in any non-specific manner. The fluorescence level of the loxP related recombination reaction was very low and was almost the same as that of the negative control. This low fluorescence level seems to be caused by the excessive intramolecular excision reactions between two identical recognition sequences. In contrast, the increased fluorescence levels were significantly high in the recombinations related to $m 2$ and lox 2272 spacer mutants. The ratios of total fluorescence were $9.8 \%$ and $7.7 \%$ compared to that of the positive control in $m 2$ and lox 2272 related recombination reactions, respectively. The total amount of fluorescence, or the recombination efficiency, in the recombination related to $m 2$ was 1.3 times higher than that of the recombination related to lox2272. Although this data is consistent with the results of the incompatibility test, the extent of difference in the recombination efficiency

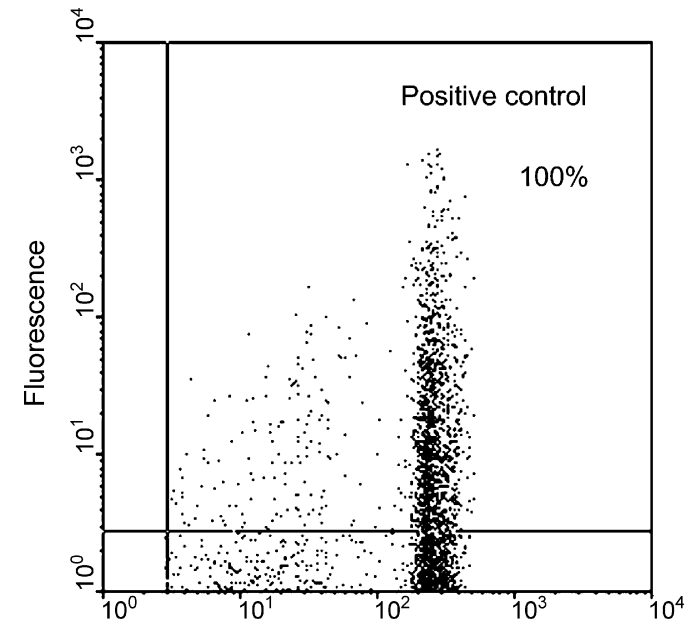

(A)

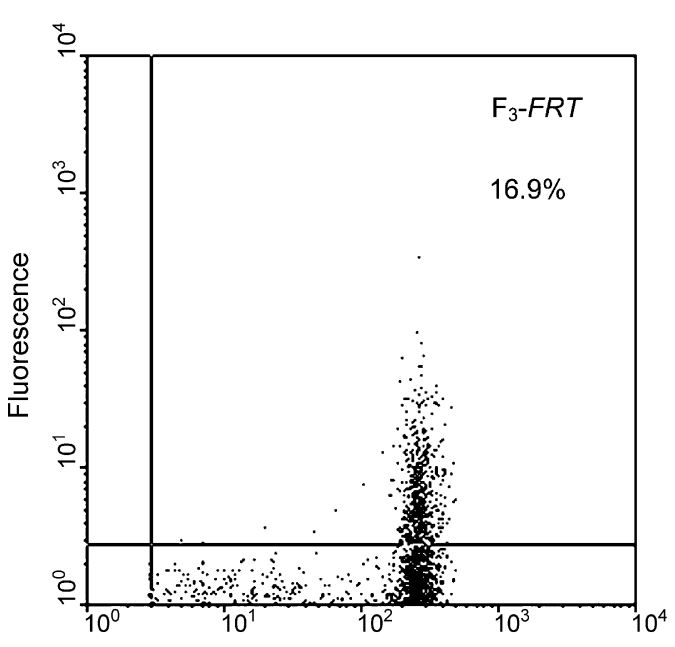

(C)

Cell size

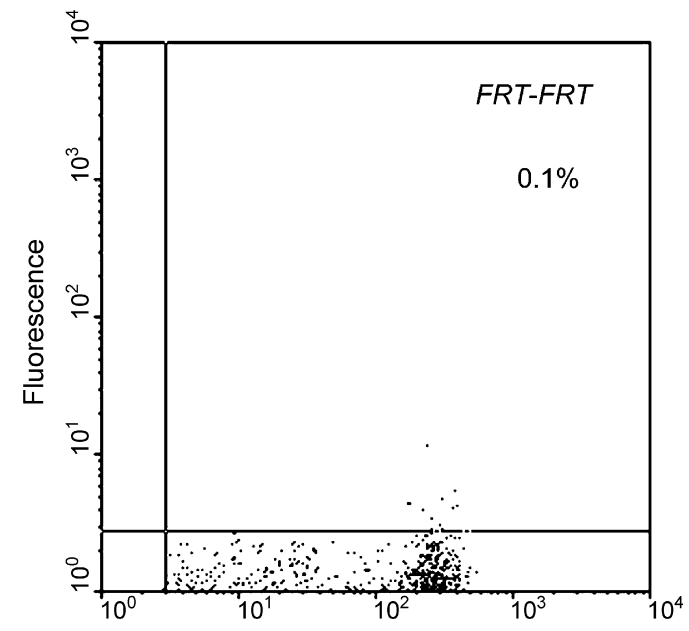

(B)

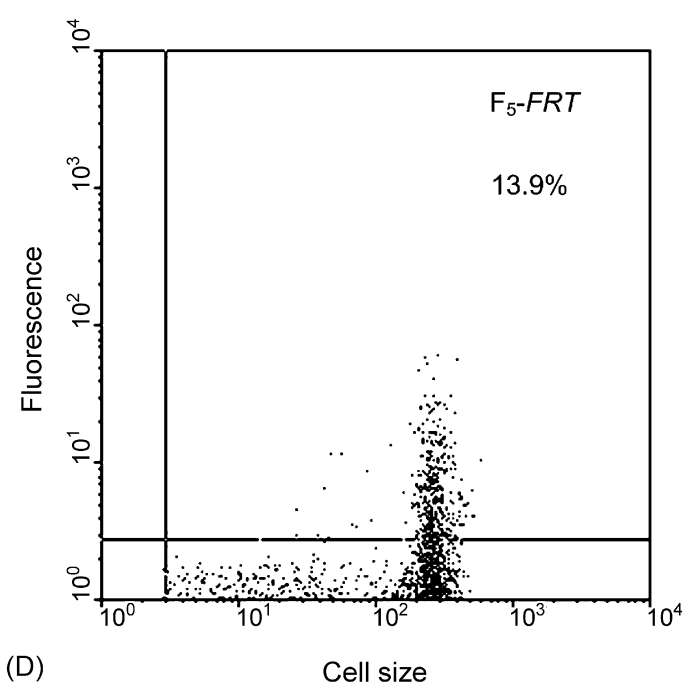

Fig. 7. FACS analysis of the recombination efficiency test in the Flpe-mediated cassette exchange. The ratio of total fluorescence of recombinations related to $F R T(\mathrm{~B}), \mathrm{F}_{3}(\mathrm{C})$, and $\mathrm{F}_{5}$ (D) compared to that of the positive control (A) were estimated. 
between $m 2$ and lox2272 related recombination reactions is higher than that of their incompatibility. From this result, it can be postulated that the sequence of the $m 2$ spacer region is more susceptible to the recombination process, such as a DNA strand exchange, than that of the lox 2272 spacer region.

Fig. 7 shows the levels of recombination efficiency in Flpe-mediate cassette exchange, which contains different aspects compared to that of Cre-mediated cassette exchange. The fluorescence level in the FRT related recombination reaction was very low, as was expected. In contrast, the increased fluorescence levels were significantly high in the recombination related to the $\mathrm{F}_{3}$ and $\mathrm{F}_{5}$ spacer mutants. The ratios of total fluorescence were $16.9 \%$ and $13.9 \%$ compared to that of the positive control in $\mathrm{F}_{3}$ and $\mathrm{F}_{5}$ related recombination reactions, respectively. The total amount of fluorescence in the recombination related to $\mathrm{F}_{3}$ was 1.2 times higher than that of the recombination related to $\mathrm{F}_{5}$. From the repetitive recombination experiments, it was concluded that the $\mathrm{F}_{3}$ mutant can mediate more efficient site-specific recombination than the $\mathrm{F}_{5}$ mutant despite its lower level of incompatibility with the FRT sequence than $\mathrm{F}_{5}$. This result can be explained by the fact that the modified sequence of the spacer region in a mutant affects not only the reactivity upon a wild-type sequence but also the recombination efficiency (Lee and Saito, 1998). The relative fluorescence levels compared to that of the positive control in various spacer mutants related recombination reactions are shown in Fig. 8.

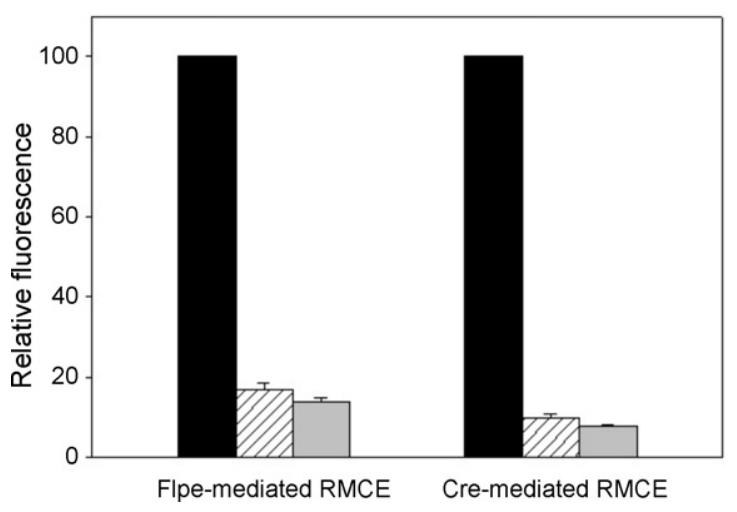

Fig. 8. Relative fluorescence of recombinations related to mutant sequences compared to the positive control (black bar). $\mathrm{F}_{3}$ and $m 2$ (dashed bar) could mediate slightly more efficient site-specific recombination than $\mathrm{F}_{5}$ and lox2272 (gray bar).
Although absolute incompatibility with wild-type sequence is the most important factor of consideration to identify mutants for efficient recombination, the efficiencies of recombinations related to various mutant candidates, which have a high level of incompatibility, eventually have to be estimated in vivo. Considering this point, our analysis system can be highlighted due to its simplicity, accuracy and applicability to any mutant sequence.

\subsection{PCR analysis}

PCR analysis was performed to confirm that recombination processes were mediated in a site-specific manner using different sizes of exchange cassettes. Primers were designed to have an exact homology with pDC family vectors. The sizes of the exchange cassettes were also adjusted to be different. The pDC family vector has an exchange cassette of about $1.6 \mathrm{~kb}$. After precise RMCE, this fragment is replaced by an exchange cassette of about $0.9 \mathrm{~kb}$ which comes from the pIC family vector (Fig. 5).

Fig. 9 shows the result of PCR analysis on Flpemediated recombination reactions. In the negative control (lane 1) and the three mixtures of pIC and pDC family vectors (lanes 3,5 and 7), there were no DNA bands approaching $0.9 \mathrm{~kb}$. And at the same time, no band approaching $0.9 \mathrm{~kb}$ was detected in the mixture of pIC$F R T$-EGFP- $F R T$, pDC- $F R T$-TK- $F R T$, and $\mathrm{p} \triangle \mathrm{EGFP}-$ Flpe (lane 4). However, the band was detected in the positive control (lane 2) and the two mixtures of pIC family vectors, $\mathrm{pDC}$ family vectors, and $\mathrm{p} \triangle \mathrm{EGFP}-$

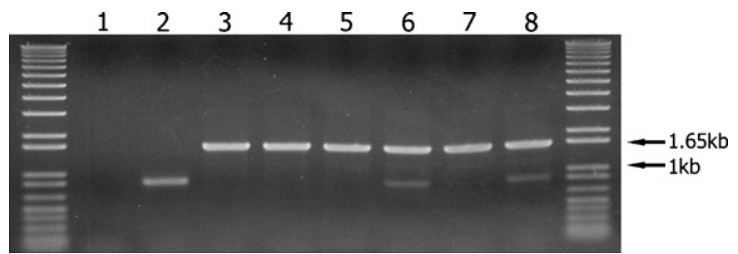

Fig. 9. PCR analysis of the Flpe-mediated recombination reactions. Lane 1, negative control; lane 2, positive control; mixture of pIC$F R T$-EGFP-FRT, pDC- $F R T$-TK- $F R T$ without $\mathrm{p} \triangle \mathrm{EGFP}-F l p e$ (lane 3 ) and with $\mathrm{p} \Delta \mathrm{EGFP}-\mathrm{Flpe}$ (lane 4); mixture of pIC-F 3 -EGFP$F R T$, pDC-F $3-T K-F R T$ without $\mathrm{p} \triangle \mathrm{EGFP}-\mathrm{Flpe}$ (lane 5) and with $\mathrm{p} \triangle$ EGFP-Flpe (lane 6); mixture of pIC-F5-EGFP- $F R T$, pDC-F 5 -TK$F R T$ without $\mathrm{p} \triangle$ EGFP-Flpe (lane 7) and with $\mathrm{p} \triangle$ EGFP-Flpe (lane 8). Complete cassette exchange by site-specific recombination results in the DNA band approaching $0.9 \mathrm{~kb}$. 
Flpe (lanes 6 and 8). In both reactions, the DNA band was extracted and cloned into a T-vector (RBC, Taipei, Taiwan). Ten colonies, from each reaction, were harvested and sequenced by M13 forward and reverse primers. In all sequencing data, reunited complete mutant sequences $\left(\mathrm{F}_{3}\right.$ and $\left.\mathrm{F}_{5}\right)$ and wild-type sequence were confirmed. The EGFP coding region was also identified. This implies that recombination reactions were mediated in a site-specific manner.

The elongation time of PCR was also increased to $5 \mathrm{~min}$ to investigate whether or not intermediates exist. Theoretically, two forms of intermediate are possible when only one reciprocal crossover occurs between mutant sequences or wild-type sequences (Fig. 5). The expected size of the DNA band is about $5 \mathrm{~kb}$ in both cases if the intermediates serve as templates in PCR. However, the band was not detected by PCR analysis. It seems that almost all intermediates disappeared because those that contain two sets of recognition targets are susceptible to further recombination and, also, an intramolecular reaction is much more likely to occur than an intermolecular reaction.

In this study, a simple FACS analysis system was proposed to evaluate the efficiency of recombination related to various mutant sequences. We selected $m 2$ and lox2272 mutant sequences for Cre-mediated cassette exchange, and $F_{3}$ and $F_{5}$ mutant sequences for Flpe-mediated cassette exchange and thereafter estimated the recombination efficiencies. There were no prominent differences in recombination efficiencies between $m 2$ and lox2272, and $F_{3}$ and $F_{5}$ mutant sequences, as was expected from their very similar and high incompatibilities with wild-type sequences. However, slight differences could be detected repeatedly and accurately using our FACS analysis system.

In conclusion, the feasibility of the FACS analysis system for the estimation of recombination efficiency was verified. Our system should be readily applicable for estimating recombination efficiency of any mutant candidate, which will contribute to more precise and efficient site-specific recombination.

\section{Acknowledgments}

This research was supported in part by grants from the Ministry of Commerce, Industry, and Energy and
Daejon city (Bio/RIS program) and the Ministry of Education (Brain Korea 21 Program).

\section{References}

Albert, H., Dale, E.C., Lee, E., Ow, D.W., 1995. Site-specific integration of DNA into wild-type and mutant lox sites placed in the plant genome. Plant J. 7, 649-659.

Arad, U., 1998. Modified Hirt procedure for rapid purification of extrachromosomal DNA from mammalian cells. BioTechniques 24, 760-762.

Baer, A., Bode, J., 2001. Coping with kinetic and thermodynamic barriers: RMCE, an efficient strategy for the targeted integration of transgenes. Curr. Opin. Biotechnol. 12, 473480.

Bell, A.C., Felsenfeld, G., 1999. Stopped at the border: boundaries and insulators. Curr. Opin. Genet. Dev. 9, 191-198.

Branda, C.S., Dymecki, S.M., 2004. Talking about a revolution: the impact of site-specific recombinases on genetic analyses in mice. Dev. Cell. 6, 7-28.

Buchholz, F., Angrand, P.O., Stewart, A.F., 1998. Improved properties of FLP recombinase evolved by cycling mutagenesis. Nat. Biotechnol. 16, 657-662.

Festenstein, R., Tolaini, M., Corbella, P., Mamalaki, C., Parrington, J., Fox, M., Miliou, A., Jones, M., Kioussis, D., 1996. Locus control region function and heterochromatin-induced position effect variegation. Science 271, 1123-1125.

Hoess, R.H., Ziese, M., Sternberg, N., 1982. P1 site-specific recombination: nucleotide sequence of the recombining sites. Proc. Natl. Acad. Sci. 79, 3398-3402.

Kolot, M., Meroz, A., Yagil, E., 2003. Site-specific recombination in human cells catalyzed by the wild-type integrase protein of coliphage HK022. Biotechnol. Bioeng. 84, 5660.

Langer, S.J., Ghafoori, A.P., Byrd, M., Leinwand, L., 2002. A genetic screen identifies novel non-compatible loxP sites. Nucleic Acids Res. 30, 3067-3077.

Lee, G., Saito, I., 1998. Role of nucleotide sequences of $\operatorname{lox} \mathrm{P}$ spacer region in Cre-mediated recombination. Gene 216, 5565 .

Lee, J., Jayaram, M., 1995. Role of partner homology in DNA recombination. J. Biol. Chem. 270, 4042-4052.

McLeod, M., Craft, S., Broach, J.R., 1986. Identification of the crossover site during FLP-mediated recombination in the Saccharomyces cerevisiae plasmid 2 microns circle. Mol. Cell. Biol. 6, 3357-3367.

Park, M.T., Lee, G.M., 2000. Rapid titer assay of adenovirus containing green fluorescent protein gene using flow cytometric analysis. Bioprocess Eng. 22, 403-406.

Ringrose, L., Lounnas, V., Ehrlich, L., Buchholz, F., Wade, R., Stewart, A.F., 1998. Comparative kinetic analysis of FLP and Cre recombinases: mathematical models for DNA binding and recombination. J. Mol. Biol. 284, 363-384.

Schlake, T., Bode, J., 1994. Use of mutated FLP recognition target $(F R T)$ sites for the exchange of expression cas- 
settes at defined chromosomal loci. Biochemistry 33, 1274612751.

Umlauf, S.W., Cox, M.M., 1988. The functional significance of DNA sequence structure in a site-specific genetic recombination reaction. EMBO J. 7, 1845-1852.
Yoon, Y.G., Posfai, G., Szybalski, W., Kim, S.C., 1998. Cre/loxPmediated in vivo excision of large segments from yeast genome and their amplification based on the 2 micron plasmid-derived system. Gene 223, 67-76. 